To appear in January 10, 1997 ApJ (Letters).

\title{
Extremely Rapid Variations of Water Maser Emission from the Circinus Galaxy
}

\author{
L. J. Greenhill ${ }^{1}$, S. P. Ellingsen ${ }^{2}$, R. P. Norris ${ }^{3}$, R. G. Gough ${ }^{3}$, M. W. Sinclair ${ }^{3}$, \\ J. M. Moran ${ }^{1}$, R. Mushotzky ${ }^{4}$
}

\begin{abstract}
The water maser lines in the Seyfert nucleus of the Circinus galaxy vary on time scales as short as a few minutes. The amplitude of one line more than doubled in $\approx 10$ minutes, reaching $\approx 37 \mathrm{Jy}$, which corresponded to an increase of $\approx 6 \mathrm{~L}_{\odot}$, assuming isotropic emission, in a single maser feature on a size scale of about $1 \mathrm{AU}$, based on light-travel time. Other lines vary by up to about $30 \%$ on similar time scales. The variability is at least two orders of magnitude more rapid than any observed for other Galactic or extragalactic water masers. The intensity changes cannot be attributed easily to a mechanism of intrinsic fluctuations. The variability may be the result of strong interstellar diffractive scintillation along the line of sight within our Galaxy. This would be the first example of diffractive scintillation for any source at $22 \mathrm{GHz}$ and for any source other than a pulsar. However, only the very shortest timescales for interstellar scintillation, obtained from pulsar observations and scaled to $22 \mathrm{GHz}$, correspond to the observed maser variability. Alternatively, the intensity changes may be a reaction to fluctuations in compact background or radiative pump sources and thereby may be related to variability of the central engine.

The maser spectral features symmetrically bracket the systemic velocity of the galaxy, with components red- and blue-shifted by about $\pm(100-200) \mathrm{km} \mathrm{s}^{-1}$. The spectrum of the Circinus maser is similar in some respects to that of the maser in NGC 4258, which probably traces a molecular disk rotating around a supermassive object. VLBI observations could reveal whether the maser source in the heart of the Circinus galaxy is part of a similar dynamical system.
\end{abstract}

\footnotetext{
${ }^{1}$ Harvard-Smithsonian Center for Astrophysics, 60 Garden St, Cambridge, MA 02138 USA, greenhill@cfa.harvard.edu

${ }^{2}$ University of Tasmania, G.P.O. 252C, Hobart, Tasmania, 7001, Australia

${ }^{3}$ Australia Telescope National Facility, CSIRO, P.O. Box 76, Epping, NSW 2121, Australia

${ }^{4}$ NASA/Goddard Space Flight Center, Laboratory for High Energy Astrophysics, Code 666, Greenbelt, MD 20771 USA
} 
Subject headings: galaxies: individual, Circinus — galaxies: kinematics and dynamics — galaxies: nuclei — masers

\section{Introduction}

Renewed interest in extragalactic water masers has been stimulated by observations of the maser in the nucleus of NGC 4258 (Miyoshi et al. 1995). Maser emission is present at the systemic velocity of the galaxy and in satellite lines offset by about $\pm 900 \mathrm{~km} \mathrm{~s}^{-1}$. The emission arises from a thin, nearly edge-on molecular disk that is in Keplerian rotation around a massive central object (see also Moran et al. 1995). These studies have established, to high accuracy, the binding mass and the geometry of this system on sub-parsec scales.

The Circinus galaxy (CG) is a Seyfert 2 galaxy at a distance of about 4 Mpc that has long been known to harbor a water maser in its nucleus (Gardner \& Whiteoak 1982). The nucleus shows a one-sided ionization cone (Marconi et al. 1994) and bipolar radio lobes that extend about $200 \mathrm{pc}$ along the minor axis of the galaxy (Elmouttie et al. 1995). Excitation of coronal lines within the inner $10 \mathrm{pc}$ is consistent with a photoionizing central continuum source, rather than with a starburst, though there are signs of an old starburst within the innermost 4 pc (Oliva et al. 1994). A heavily obscured hard (2-10 keV) X-ray source (Matt et al. 1996) and a compact radio synchrotron source also lie in the nucleus (Norris \& Forbes 1996).

The water maser spectrum of the CG is similar to that of NGC 4258 in two ways. First, there are two complexes of narrow lines that bracket the systemic velocity of the galaxy. Second, there is a distinct complex of emission lines close to the systemic heliocentric velocity of $416 \mathrm{~km} \mathrm{~s}^{-1}$ (Lehnert \& Heckman 1995). (We use the radio definition of radial velocity throughout.) The red-shifted lines in the CG were discovered by Gardner \& Whiteoak (1982), but the blue-shifted lines have only recently been detected by us (reported in this paper), and by Nakai et al. (1995) and Braatz, Wilson, \& Henkel (1996), independently.

Whiteoak \& Gardner (1986) observed that the shape of the CG maser spectrum varies substantially in about one month. The relative intensities of spectral components in the NGC 4258 maser can also vary by tens of percent in a few weeks (Greenhill et al. 1995). The maser in IC 10 can vary by $60 \%$ in less than one day (Argon et al. 1994), which may be a consequence of interstellar scattering. We note that the most rapid known flares in Galactic interstellar $\mathrm{H}_{2} \mathrm{O}$ masers have time scales of one to ten days (e.g., Liljeström 1993; Pacheco \& Scalise 1993; Haschick, Burke, \& Spencer 1977). In this Letter, we report the measurement of a finely sampled time-series of spectra, which has revealed variability on time scales at least two orders of magnitude shorter than those known for any other water maser. We also analyze the morphology of these spectra in the context of an edge-on disk geometry. 


\section{Observations}

The observations were carried out at the Australia Telescope National Facility's Parkes radio telescope in 1995 October, as part of a search for new water maser sources in 28 galaxies $(z<0.1)$ with high X-ray absorption columns or excess infrared emission accompanied by compact radio continuum emission. We used a newly commissioned $22 \mathrm{GHz}$ receiver, with two orthogonal linear polarization channels. From a tipping scan, we estimate that the receiver temperature was $95 \mathrm{~K}$, and that the atmospheric opacity at zenith was 0.06 . The telescope sensitivity was about $5.7 \mathrm{Jy}$ $\mathrm{K}^{-1}$, based on a flux density of $21.2 \mathrm{Jy}$ for Virgo A. Uncertainties in pointing are responsible for up to $10 \%$ loss in gain. We adopted the gain curve of Bourke (1994), increased by about $10 \%$ at low elevation angles as indicated by our observations of Virgo A. We observed in position-switching mode, with four $16 \mathrm{MHz}$ bandpasses tuned to cover the range of emission from $195-735 \mathrm{~km} \mathrm{~s}^{-1}$. The 4096-channel digital autocorrelator provided a channel spacing of $15.625 \mathrm{kHz}$, or $0.21 \mathrm{~km} \mathrm{~s}^{-1}$.

\section{The observed spectrum and variability}

The spectrum of the CG water maser shows two complexes of strong lines between 510 and $610 \mathrm{~km} \mathrm{~s}^{-1}$, and between 240 and $310 \mathrm{~km} \mathrm{~s}^{-1}$ (see Fig. 1). We refer to these as the red and the blue satellite lines. Spectra taken in previous years show similar velocity ranges for the complexes of satellite lines (Whiteoak \& Gardner 1986; Nakai et al. 1995). Figure 2 shows the relative flux density variations for the three strongest maser lines on time scales as short as a few minutes. The $567.25 \mathrm{~km} \mathrm{~s}^{-1}$ feature more than doubled in strength to about 37 Jy in only 10 minutes between 15 and 16 hours LMST on day 289. On the assumption of isotropic emission this represents an increase from 4.8 to $11 \mathrm{~L}_{\odot}$. At other times the strength of this feature varies by about $50 \%$, while the other two features vary upto about $30 \%$, on similar time scales. We conservatively adopt a characteristic time scale for variability, $t_{o b s}$, of $\sim 300 \mathrm{~s}$.

We feel certain that instrumental effects are not responsible for the apparent maser fluctuations. The variations of different spectral features are not correlated. Moreover, observations of the CG and NGC 4945 masers were made within hours of each other, and no variability was detected in NGC 4945. Apparent variability might be a consequence of telescope pointing errors if the maser features were distributed over an area comparable to the 1'.3 primary beam. However, spectra taken at offset positions show that the region of maser emission is relatively compact, $<0.3$, as expected for an extragalactic nuclear maser. False variability might also arise from under-sampling of the spectral lines but in our case the half-power widths (FWHM) of the maser features are at least four spectral channels.

The FWHM of the narrowest satellite spectral line is about $0.9 \mathrm{~km} \mathrm{~s}^{-1}$. The satellite lines seen in NGC 4258 are about $1.0 \mathrm{~km} \mathrm{~s}^{-1}$ wide (Miyoshi et al. 1995), and the narrowest water maser lines ever observed are about $0.3 \mathrm{~km} \mathrm{~s}^{-1}$ in IC 10 (Argon et al. 1994). Emission from masers in the presence of a magnetic field may be linearly polarized (Watson 1994a). We used our dual 


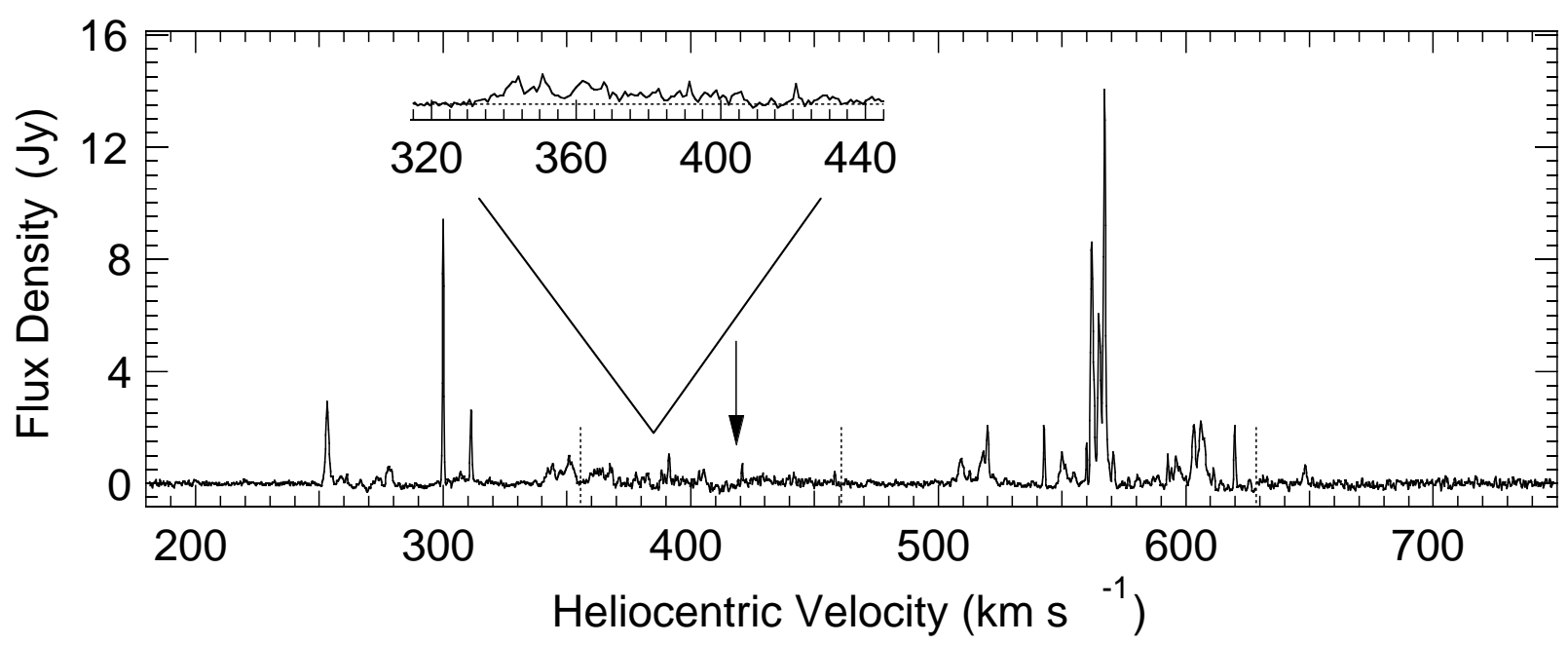

Fig. 1. - Time-averaged spectrum of the water maser emission from the nucleus of the Circinus galaxy. The heliocentric systemic velocity of the galaxy, $416 \mathrm{~km} \mathrm{~s}^{-1}$, is indicated by the arrow. The dashed lines indicate the divisions between the four receiver bandpasses. The on-source integration time for the two bandpasses that contain the two strong complexes of lines was 350 minutes ( $1 \sigma$ noise $=0.05 \mathrm{Jy})$. The integration time for the other bands was 100 minutes $(1 \sigma$ noise $=0.1$ Jy). The spectrum was Hanning smoothed and has a channel spacing of $0.21 \mathrm{~km} \mathrm{~s}^{-1}$. The three spectral features examined in Figure 2 are indicated by the bars along the top axis. (insert) Spectrum obtained separately that shows more clearly the emission near the systemic velocity (as a consequence of better baseline removal). The on-source integration time was 60 minutes and the channel spacing was $0.8 \mathrm{~km} \mathrm{~s}^{-1}$. The gain calibration is accurate to $\approx 10 \%$ and includes corrections for atmospheric attenuation, antenna gain variations, and average pointing error.

polarization observations of the satellite lines to construct Stokes Q spectra of the source. There is no statistically significant linear polarization at a level of $0.5 \mathrm{Jy}$ ( $4 \%$ for a $12 \mathrm{Jy}$ line).

\section{Origins of Variability}

The maser variability is extraordinary because a light travel time of a few minutes implies a characteristic scale of about 1 AU. Masers may be visualized typically as filamentary structures, viewed end-on, which mark local enhancements in density, velocity coherence, or excitation conditions (Elitzur 1992). Large fluctuations in emission rate are caused by changes in the maser medium over lengths greater than the maser gain length, $\ell$, which is the characteristic length over which the line-center opacity changes by $\sim 1$. For typical conditions, $\ell \gg 1$ AU (e.g., a maser pump efficiency of $1 \%$, a fractional water abundance of $10^{-4}$, and a temperature of $400 \mathrm{~K}$ as in Reid \& Moran 1988). To be responsible for the observed maser variability, changes in the maser medium must propagate at about the speed of light, and we know of no suitable driving mechanism. 


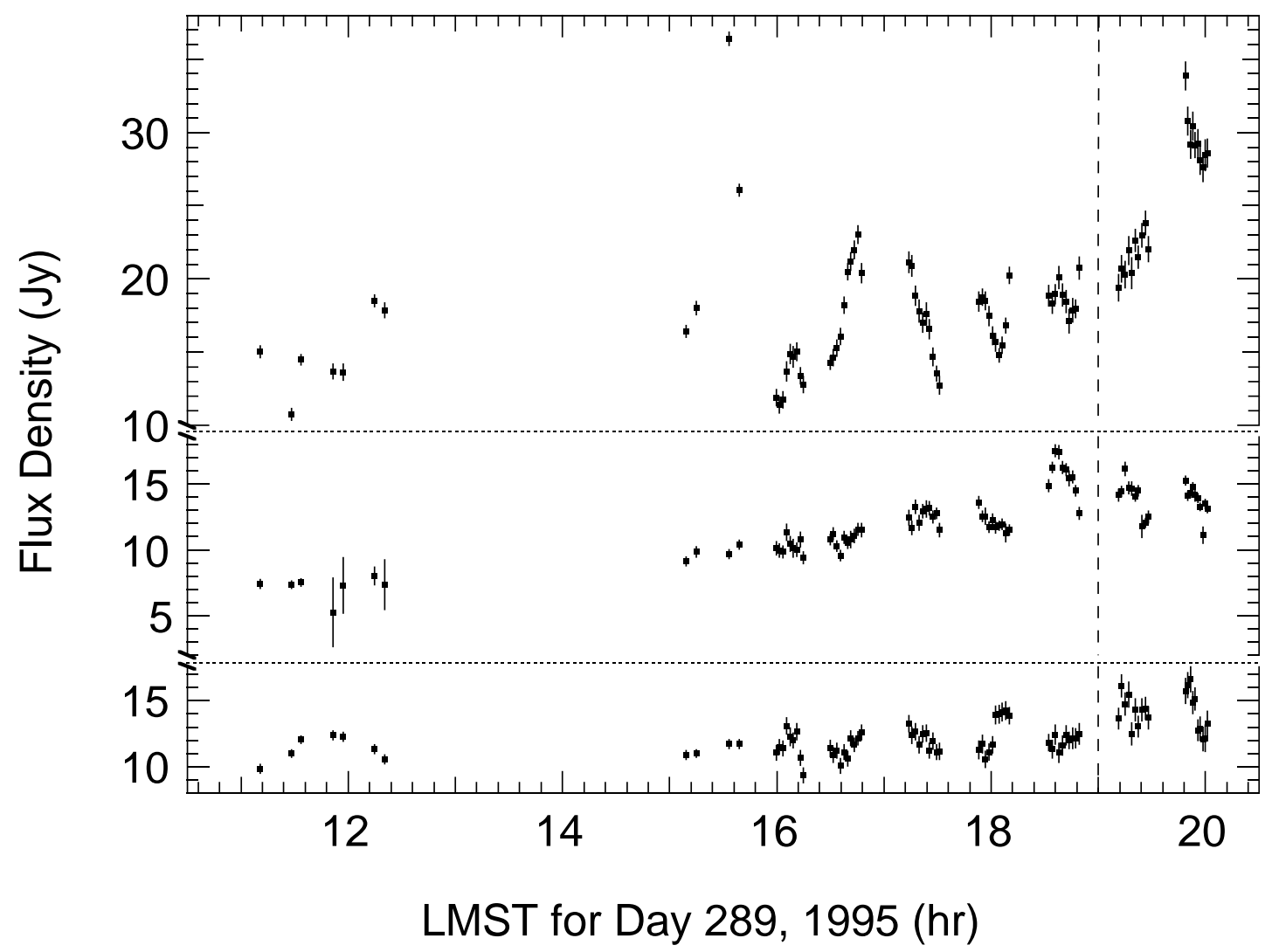

Fig. 2.- Radio light curves for three of the strongest spectral components, (top) 567.25, (middle) 562.00 , and (bottom) $299.91 \mathrm{~km} \mathrm{~s}^{-1}$. Instrumental effects cannot be responsible for the time variability. Before 16 LMST data were taken in 5 minute integrations, and after 16 LMST in 1 minute integrations. Data gaps occurred when the telescope was off source. The error bars reflect the formal uncertainties from fitting Gaussian line profiles to the spectra. It is possible that an error in the calibration at the lowest elevations causes a slow upward trend in flux densities after 19 LMST, on the order of $10 \%$.

There are three mechanisms by which the maser may vary in response to processes external to the maser medium: interstellar scintillation, amplification of a time-variable background source, and excitation by a time-variable radiative pump source. The last two mechanisms assume the presence of a compact (1 AU) source in the vicinity of the maser. Large amplitude fluctuations on time scales close to $10^{3} \mathrm{~s}$ have been observed towards nuclei at X-ray energies (Mushotzky, Done, \& Pounds 1993), as in NGC 4051 (Guainazzi et al. 1996) and Mrk 766 (Leighly et al. 1996).

Interstellar scintillation (ISS) is caused by scattering in an ionized component of the Galactic interstellar medium, which may be important because the CG lies at a Galactic latitude of only $-3.8^{\circ}$ (longitude $311^{\circ}$ ). We consider scintillation caused by a thin, turbulent scattering screen at distance $D$ that is moving with respect to the observer at velocity $v$. The turbulence is usually 
described by a Kolmogorov power law Armstrong, Cordes, \& Rickett 1981) and the scintillation is divided into two regimes, weak and strong. For a point source, the time scale of weak ISS is approximately the crossing time of the Fresnel scale, $\ell_{F} \approx \sqrt{D \lambda / 2 \pi}$, where $\lambda$ is wavelength (Narayan 1993). For $D=1 \mathrm{kpc}, \lambda=1.35 \mathrm{~cm}$, and $v=100 \mathrm{~km} \mathrm{~s}^{-1}, \ell_{F} \approx 2.5 \times 10^{10} \mathrm{~cm}$ and $t_{F}=\ell_{F} / v \approx 4000 \mathrm{~s}$ (Rickett 1990), about an order of magnitude greater than $t_{o b s}$. For strong ISS the contributing turbulent length scales obey $\ell_{\text {scin }}<\ell_{F}$. (Strong scintillation may be diffractive or refractive. We do not consider the second because the relevant length scales are longer.) Diffractive scintillation at $1 \mathrm{GHz}$ with time scales of $t_{\text {scin }}>20 \mathrm{~s}$ is observed toward pulsars for which $D>1$ $\mathrm{kpc}$ and $2^{\circ}<b<5^{\circ}$ (Cordes 1986). For Kolmogorov turbulence, $t_{\text {scin }} \propto \nu^{1.2}$, where $\nu$ is frequency. Goodman \& Narayan (1985) show that for non-Kolmogorov scattering screens $t_{\text {scin }} \propto \nu^{1.0}$. In either case, only the fastest observed scintillation at $1 \mathrm{GHz}, t_{\text {scin }} \sim 10 \mathrm{~s}$, corresponds to sufficiently rapid variations at $22 \mathrm{GHz}$. However, the true angular size of a scintillating maser feature, $\theta_{m}$, must also be less than $\theta_{\text {scin }}=\ell_{\text {scin }} / D$ because otherwise the scintillation is slowed by a factor of $\left(\theta_{m} / \theta_{\text {scin }}\right)$ and the amplitude is similarly decreased (Narayan 1993). Hence, for strong scintillation, $\theta_{m}<\theta_{\text {scin }}<\theta_{F}$, which corresponds to $<7 \mathrm{AU}$ at a distance of $4 \mathrm{Mpc}$, where $\theta_{F}=\ell_{F} / D$. The scintillation time scale for a scattering screen in the AGN, within $<1 \mathrm{kpc}$ of the maser, may be $<t_{o b s}$, but the amplitude of the variations will be substantially reduced because the angular size of the maser source, viewed from the screen, will be large.

The time scale of variability for a maser that amplifies a background source is not limited by the extent of the maser. The maser medium forms an image of the background source, and the angular sizes of the two are the same (see Haschick et al. 1990). For the CG, the background source must be less than a few light-minutes in size, and the apparent maser cross-section would be about $0.25 \mu$ as or $1 \mathrm{AU}$. We note that substantial variability may be possible only if a fraction of the maser is unsaturated since the emission rate of a completely saturated maser is fixed by the pump rate and beam angle. In this case, the beam angle is set by the solid angle of the background source as seen from the maser (see Haschick et al. 1990).

The variability time scale of a radiatively pumped maser is also independent of its physical extent, apart from optical-depth effects. The pump luminosity, which may be supplied directly by the central engine, must be large enough that the pump rate exceeds the maser emission rate. For Galactic interstellar water masers, radiative pumping by stellar radiation generally cannot support observed emission rates however, substantially higher luminosities are present in AGN. For a single pump transition (at wavelength $\left.\lambda_{p}\right), F_{\nu p}>F_{\nu m}\left(\Omega_{b} / \Omega_{m}\right)\left(x_{m} / x_{p}\right)$, where $F_{\nu p}$ and $F_{\nu m}$ are the pump and maser flux densities, respectively, $\Omega_{b}$ is the maser beam solid angle, $\Omega_{m}$ is the solid angle of the maser viewed from the pump, and $x_{p}$ and $x_{m}$ are the fractional bandwidths of the pump and maser, which are similar (Reid \& Moran 1988). For $N$ simultaneous pump transitions the requisite $F_{\nu p}$ is reduced proportionately. We assume the central engine has a flat spectral energy distribution $\left(\nu F_{\nu}\right)$ between ultraviolet and X-ray energies and estimate $F_{\nu p}$ at $\lambda_{p}$ from the inferred intrinsic X-ray luminosity of the central engine. Matt et al. (1996) obtain a luminosity of $4 \times 10^{41} f^{-1} \mathrm{erg} \mathrm{s}^{-1}$ from the observed X-ray spectrum, assuming that a fraction, 
$f=\left(\Omega_{R} / 2 \pi\right)$, is reflected by a portion of the obscuring circumnuclear torus into the line of sight, where $\Omega_{R}$ is the solid angle of the reflecting material seen from the central engine. Since the infrared luminosity of the nucleus is $\approx 5 \times 10^{43} \mathrm{erg} \mathrm{s}^{-1}$ (Moorwood \& Glass 1984), $f$ is on the order of a few percent. Based on order of magnitude estimates for the parameters involved, radiative pumping requires that $\lambda_{p} \sim 0.6 N^{-1}\left(\frac{\Omega_{b}}{\Omega_{m}}\right)\left(\frac{F_{\nu m}}{10 \mathrm{Jy}}\right)\left(\frac{f}{0.01}\right)\left(\frac{D}{4 \mathrm{Mpc}}\right)^{2}$, where $\lambda_{p}$ is expressed in units of microns, and $\left(\frac{\Omega_{b}}{\Omega_{m}}\right) \sim 1$ (Reid \& Moran 1988). Hence, a radiative maser pump may be feasible if it consists of on the order of 10 transitions at ultraviolet wavelengths.

Several other specific mechanisms for maser variability have been proposed in the literature, though none of them explains the observed time scale. First, in partially saturated masers, a radiative instability can cause variations with a period of $L / c$, where $L$ is the maser filament length (Scappaticci \& Watson 1992). However, strong maser emission requires many gain lengths or $L / c \gg t_{\text {obs }}$. Second, masers may amplify each other, and an outburst can occur when one maser drifts in front of another with the same line-of-sight velocity (Deguchi \& Watson 1989). Third, rotation of a maser beam across the line of sight can mimic rapid intensity variations Norris \& Booth 1981). However, for the latter two mechanisms, maser lengths of $\gg 1 \mathrm{AU}$ and transverse velocities of $<100 \mathrm{~km} \mathrm{~s}^{-1}$ require maser cross-sections of $<10^{10} \mathrm{~cm}$, which are improbably small.

\section{Structure of the maser source}

Based on the morphology of the $\mathrm{H}_{2} \mathrm{O}$ maser spectrum, we propose that the maser emission arises from a nearly edge-on Keplerian disk, as in NGC 4258. The satellite lines arise on a disk diameter perpendicular to the line of sight, and the systemic emission arises on the near side of the disk, close to the line of sight to the dynamical center. These locations naturally possess the greatest line-of-sight velocity coherence. For Keplerian rotation, the outer radius of the disk is about four times the inner radius because the velocities of the satellite lines at these radii, relative to the systemic velocity, differ by a factor of two (i.e., 100 and $200 \mathrm{~km} \mathrm{~s}^{-1}$, respectively). The binding mass for the disk in solar units is $10^{6} r_{0.1} \sin ^{2} i$, where $r_{0.1}$ is the disk inner radius in units of $0.1 \mathrm{pc}$, and $i$ is the inclination of the disk rotation axis. A central engine of $<10^{7} \mathrm{M}_{\odot}$, with a gravitational radius of $<3 \times 10^{12} \mathrm{~cm}$, can vary on time scales of $100 \mathrm{~s}$ (cf., Mushotzky et al. (1993)), as may be required by the observed maser variability. We note that for rotation speeds of $200 \mathrm{~km} \mathrm{~s}^{-1}$ and $r_{0.1}>0.04$, the line-of-sight centripetal acceleration of systemic maser features in the spectrum will be $<1 \mathrm{~km} \mathrm{~s}^{-1} \mathrm{yr}^{-1}$ (cf., Greenhill et al. 1995).

\section{Conclusions}

We have observed extremely rapid variability in the spectrum of the nuclear water maser in the Circinus galaxy. In principle, fluctuations on time scales of a few minutes may be explained by ISS in our Galaxy, variability of background emission that is being amplified, or variability 
of a radiative pump. While only the shortest measured scintillation time scales for pulsars, extrapolated to $22 \mathrm{GHz}$, are acceptable, the sensitivity limits of the pulsar observations may hide still more rapid diffractive ISS. In addition, the scattering medium at low latitude is clumpy, which may also allow more rapid scintillation along the particular line of sight to the CG. If the maser fluctuations are caused by diffractive ISS, then the maser is the first example for any source at $22 \mathrm{GHz}$ and for any source other than a pulsar. We note that the only other extragalactic water maser source reported to display significant variations over $<1$ day (IC 10) also lies at low latitude, $3^{\circ}$ (Argon et al. 1994).

Since the maser spectrum consists of independently varying components, the alternative background amplification and radiative pump schemes require either several compact fluctuating sources or variable pump illumination that depends on location or Doppler velocity. Nonetheless, if the CG maser directly reflects the intrinsic variability of the central engine on time scales of a few minutes, then the maser will be a powerful probe of this heavily obscured AGN. Rapid variability has been observed exclusively in X-ray wavebands for other AGN but this does not necessarily preclude the possibility that emission from central engines also varies about as rapidly at other wavelengths.

Scintillation and background amplification models place stringent upper limits of $1-10 \mathrm{AU}$ on the apparent sizes of the maser components, which implies very large brightness temperatures, $>10^{16} \mathrm{~K}$. The general symmetry of the maser spectrum with respect to the systemic velocity is similar to that of NGC 4258, in which the masers lie in a Keplerian molecular disk bound by a massive central engine. In the absence of VLBI data, we propose that the same model may be applicable to the CG maser. The highly ordered velocity field of a thin Keplerian disk naturally supports highly elongated masers, which Watson (1994b) argues is a necessary condition to permit the inferred high brightness temperatures

We thank D. Backer, C. Gwinn, R. Manchester, R. Narayan, and W. Watson, for helpful discussions. We are also grateful to Euan Troup, Harry Fagg, and Warwick Wilson for their special assistance at Parkes.

\section{REFERENCES}

Argon, A. L., Greenhill, L. J., Moran, J. M., Reid, M. J., Menten, K. M., Henkel, C., \& Inoue, M. 1994, ApJ, 422, 586

Armstrong, J. W., Cordes, J. M., \& Rickett, B. J. 1981, Nature, 291, 561

Bourke, T. L., 1994, MSc thesis, University of New South Wales

Braatz, J. A., Wilson, A. S., \& Henkel, C. 1996, ApJS, in press 
Cordes, J. M. 1986, ApJ, 311, 183

Deguchi, S. \& Watson, W. D. 1989, ApJ, 340, L17

Elitzur, M. 1992, “Astronomical Masers,” (Dordrecht: Kluwer), 155ff

Elmouttie, M., Haynes, R. F., Jones, K. L., Ehle, M., Beck, R., \& Wielebinski, R. 1995, MNRAS, 275, L53

Gardner, F. F., \& Whiteoak, J. B. 1982, MNRAS, 201, 13p

Goodman, J. \& Narayan, R. 1985, MNRAS, 214, 519

Greenhill, L. J., Henkel, C., Becker, R., Wilson, T. L., \& Wouterloot, J. G. A. 1995, A\&A, 304, 21

Guainazzi, M., Mihara, T., Otani, C., \& Matsuda, M. 1996, PASJ, in press

Haschick, A. D., Burke, B. F., \& Spencer, J. H. 1977, Science, 198, 1153

Haschick, A. D., Baan, W. A., Schneps, M. H., Reid, M. J., Moran, J. M., \& Güsten, R. 1990, ApJ, 356, 149

Lehnert, M. D. \& Heckman, T. M. 1995, ApJS, 97, 89

Leighly, K. M., Mushotzky, R. F, Yaqoob, T., Kunieda, H., \& Edelson, R. 1996, ApJ, in press

Liljeström, T. 1993, in "Astrophysical Masers", A. W. Clegg \& G. E. Nedoluha eds., (Berlin: Springer), 291

Marconi, A., Moorwood, A. F. M., Origlia, L., \& Oliva, E. 1994, Messenger, 78, 20

Matt, G., et al. 1996, MNRAS, 281, L69

Miyoshi, M., et al. 1995, Nature 373, 127

Moorwood, A. F. M., \& glass, I. S. 1984, A\&A135, 281

Moran, J. M., et al. 1995, Proc. Nat. Acad. Sci., 92, 11427

Mushotzky, R. F., Done, C., \& Pounds, K. A. 1993, ARA\&A, 31, 717

Nakai, N., Inoue, M., Miyazawa, K., Miyoshi, M., \& Hall, P. 1995, PASJ, 47, 771

Narayan, R. 1993, in "Pulsars as Physics Laboratories," R. D. Blandford, A. Hewish, A. G. Lyne, \& L. Mestel eds., (London: Oxford), 151

Norris, R. P., Booth, R. 1981, MNRAS, 195, 213

Norris, R. P., Forbes, D. C. 1996, in preparation 
Oliva, E., Salvati, M., Moorwood, A. F. M., \& Marconi, A. 1994, A\&A, 288, 457

Pacheco, G. M., \& Scalise, E. 1993, in "Astrophysical Masers", A. W. Clegg \& G. E. Nedoluha eds., (Berlin: Springer), 303

Pier, E. A., Antonucci, R., Hurt, T., Kriss, G., \& Krolik, J. 1994, ApJ, 428, 124

Reid, M. J., \& Moran, J. M. 1988, in "Galactic and Extragalactic Radio Astronomy," eds. G. L. Verschuur and K. I. Kellermann (New York: Springer), chap. 6

Rickett, B. J. 1990, ARA\&A, 28, 561

Scappaticci, G. A., \& Watson, W. D. 1992, ApJ, 400, 351

Watson, W. D. 1994a, ApJ, 424, L37

Watson, W. D. 1994b, in "The Structure and Content of Molecular Clouds, Lecture Notes in Physics, 439," T.L. Wilson \& K.J. Johnston eds., (Berlin: Springer-Verlag), 109

Whiteoak, J. B., \& Gardner, F. F. 1986, MNRAS, 222, 513 